\title{
New tests of variability of the speed of light.
}

\author{
Mariusz P. Da̧browski ${ }^{1,2,3, a}$, Vincenzo Salzano ${ }^{1}$, Adam Balcerzak ${ }^{1}$, and Ruth Lazkoz ${ }^{4}$ \\ ${ }^{1}$ Institute of Physics, University of Szczecin, Wielkopolska 15, 70-451 Szczecin, Poland \\ ${ }^{2}$ National Centre for Nuclear Research, Andrzeja Soltana 7, 05-400 Otwock, Poland, \\ ${ }^{3}$ Copernicus Center for Interdisciplinary Studies, Sławkowska 17, 31-016 Kraków, Poland \\ ${ }^{4}$ Fisika Teorikoaren eta Zientziaren Historia Saila, Zientzia eta Teknologia Fakultatea, \\ Euskal Herriko Unibertsitatea, 644 Posta Kutxatila, 48080 Bilbao, Spain
}

\begin{abstract}
We present basic ideas of the varying speed of light cosmology, its formulation, benefits and problems. We relate it to the theories of varying fine structure constants and discuss some new tests (redshift drift and angular diameter distance maximum) which may allow measuring timely and spatial change of the speed of light by using the future missions such as Euclid, SKA (Square Kilometer Array) or others.
\end{abstract}

\section{Introduction - main frameworks of varying constants theories}

In 1937 Paul Dirac [1] made interesting remarks about the relations between atomic and cosmological quantities bearing in mind that the gravitational constant is proportional to the Hubble parameter $G \propto H(t)=(d a / d t) / a$ and concluding that the former must evolve in time $-G(t) \propto 1 / t$, and the scale factor $a(t) \propto t^{1 / 3}$. The conclusion was to explain that gravity is "weak" compared to electromagnetism since the universe is "old" i.e. $F_{e} / F_{p} \propto\left(e^{2} / m_{e} m_{p}\right) t \propto t$, where $e$ is the charge, $m_{e}$ electron and $m_{p}$ proton mass. First fully quantitative framework of varying constant theory was proposed by Brans and Dicke [2] (scalar-tensor gravity) in which the gravitational constant $G$ was associated with an average gravitational potential (scalar field) $\phi$ surrounding a given particle: $\left\langle\phi>=G M /\left(c / H_{0}\right) \propto 1 / G=\right.$ $1.35 \times 10^{28} \mathrm{~g} / \mathrm{cm}$. In this approach the scalar field gives the strength of gravity $G=1 / 16 \pi \Phi$ and, as it emerged later, the action which reads as

$$
S=\int d^{4} x \sqrt{-g}\left(\Phi R-\frac{\omega}{\Phi} \partial_{\mu} \Phi \partial^{\mu} \Phi+\Lambda+L_{m}\right)
$$

in fact, relates also to the low-energy-effective superstring theory for $\omega=-1$ where the string coupling constant $g_{s}=\exp (\phi / 2)$ changes in time, and $\phi$ is the dilaton field related to Brans-Dicke field $\Phi=$ $\exp (-\phi)[3]$.

\section{Benefits and problems of varying $c$ theories}

Though attempts were performed already by Einstein [4], then by Dicke [5], Petit [6], and Moffat [7], the most popular approach was found by Albrecht and Magueijo [8] who introduced a scalar field

a e-mail: mpdabfz@wmf.univ.szczecin.pl 
$c^{4}=\psi\left(x^{\mu}\right), \mu=0,1,2,3$, with the action (R - Ricci scalar, $\Lambda$ - the cosmological constant, $L_{m}$ - matter, $L_{\psi}$ - scalar field)

$$
S=\int d^{4} x \sqrt{-g}\left[\frac{\psi(R+2 \Lambda)}{16 \pi G}+L_{m}+L_{\psi}\right] .
$$

This model breaks Lorentz invariance (relativity principle and light principle), so that there is a preferred frame (called cosmological or CMB frame) in which the field is minimally coupled to gravity. The Riemann tensor is computed in such a frame for a constant $c=\psi^{1 / 4}$ and no additional derivative terms of the type $\partial_{\mu} \psi$ appear in this frame (though they do in other frames). Einstein equations remain the same form, except that $c$ now varies.

The varying $c$ theories can be related to varying fine structure constant $\alpha$ (or charge $e=e_{0} \epsilon\left(x^{\mu}\right)$ ) theories $[9,10]$

$$
S=\int d^{4} x \sqrt{-g}\left(R-\frac{\omega}{2} \partial_{\mu} \psi \partial^{\mu} \psi-\frac{1}{4} f_{\mu v} f^{\mu v} e^{-2 \psi}+L_{m}\right)
$$

with $\psi=\ln \epsilon$ and $f_{\mu \nu}=\epsilon F_{\mu \nu}$ is the electromagnetic tensor. This is due to the definition of the fine structure constant

$$
\alpha\left(x^{\mu}\right)=\frac{e^{2}}{\hbar c\left(x^{\mu}\right)} .
$$

Assuming linear expansion of the field $\psi, e^{\psi}=1-8 \pi G \zeta\left(\psi-\psi_{0}\right)=1-\Delta \alpha / \alpha$ with the constraint on the local equivalence principle violence $|\zeta| \leq 10^{-3}$, we have the relation to dark energy [25, 26]:

$$
w+1=\frac{\left(8 \pi G \frac{d \psi}{d \ln a}\right)^{2}}{\Omega_{\psi}},
$$

where $w$ is the barotropic index, $\Omega_{\psi}$ is the dimensionless density parameter of the $\psi$ field. The field equations for Friedmann universes based on the action (3) are [11]

$$
\begin{aligned}
\frac{\dot{a}^{2}}{a^{2}} & =\frac{8 \pi G}{3}\left(\varrho_{r}+\varrho_{\psi}\right)-\frac{k c^{2}}{a^{2}}, \\
\frac{\ddot{a}}{a} & =-\frac{8 \pi G}{3}\left(\varrho_{r}+2 \varrho_{\psi}\right), \\
\ddot{\psi} & +3 \frac{\dot{a}}{a} \dot{\psi}=0,
\end{aligned}
$$

where $\varrho_{r} \propto a^{-4}$ stands for the density of radiation while

$$
\varrho_{\psi}=\frac{p_{\psi}}{c^{2}}=\frac{\sigma}{2} \dot{\psi}^{2}
$$

stands for the density of the scalar field $\psi$ (standard with $\sigma=+1$, and phantom with $\sigma=-1$ ) and

$$
\alpha=\alpha_{0} e^{2 \psi}
$$

Applying the simplest method, one derives Einstein-Friedmann equations generalized to varying speed of light (VSL) theories and varying gravitational constant G theories as ( $\varrho$ - mass density; $\varepsilon=\varrho c^{2}(t)$ - energy density in $\mathrm{Jm}^{-3}=\mathrm{Nm}^{-2}=\mathrm{kgm}^{-1} \mathrm{~s}^{-2}$ )

$$
\begin{aligned}
& \varrho(t)=\frac{3}{8 \pi G(t)}\left(\frac{\dot{a}^{2}}{a^{2}}+\frac{k c^{2}(t)}{a^{2}}\right), \\
& p(t)=-\frac{c^{2}(t)}{8 \pi G(t)}\left(2 \frac{\ddot{a}}{a}+\frac{\dot{a}^{2}}{a^{2}}+\frac{k c^{2}(t)}{a^{2}}\right),
\end{aligned}
$$


Table 1. The set of singularities for Friedmann geometry [15-17]

\begin{tabular}{lccccccc}
\hline & Name & $\mathrm{t}$ sing. & $\mathrm{a}\left(\mathrm{t}_{s}\right)$ & $\varrho\left(t_{s}\right)$ & $\mathrm{p}\left(\mathrm{t}_{s}\right)$ & $\dot{p}\left(t_{s}\right)$ etc. & $\mathrm{w}\left(\mathrm{t}_{s}\right)$ \\
\hline & Big-Bang (BB) & 0 & 0 & $\infty$ & $\infty$ & $\infty$ & finite \\
0 & Big-Rip (BR) & $t_{s}$ & $\infty$ & $\infty$ & $\infty$ & $\infty$ & finite \\
$\mathrm{I}$ & Little-Rip (LR) & $\infty$ & $\infty$ & $\infty$ & $\infty$ & $\infty$ & finite \\
$\mathrm{I}_{l}$ & Pseudo-Rip (PR) & $\infty$ & $\infty$ & finite & finite & finite & finite \\
$\mathrm{I}_{p}$ & Sudden Future (SFS) & $t_{s}$ & $a_{s}$ & $\varrho_{s}$ & $\infty$ & $\infty$ & finite \\
$\mathrm{II}$ & Gen. Sudden Future (GSFS) & $t_{s}$ & $a_{s}$ & $\varrho_{s}$ & $p_{s}$ & $\infty$ & finite \\
$\mathrm{II}_{g}$ & Finite Scale Factor (FSFS) & $t_{s}$ & $a_{s}$ & $\infty$ & $\infty$ & $\infty$ & finite \\
$\mathrm{III}$ & Big-Separation (BS) & $t_{s}$ & $a_{s}$ & 0 & 0 & $\infty$ & $\infty$ \\
$\mathrm{IV}$ & W-singularity (w) & $t_{s}$ & $a_{s}$ & 0 & 0 & 0 & $\infty$ \\
$\mathrm{V}$ & & & & & & & \\
\hline
\end{tabular}

and the generalized conservation law is obtained from (11) and (12) as

$$
\dot{\varrho}(t)+3 \frac{\dot{a}}{a}\left(\varrho(t)+\frac{p(t)}{c^{2}(t)}\right)=-\varrho(t) \frac{\dot{G}(t)}{G(t)}+3 \frac{k c(t) \dot{c}(t)}{4 \pi G a^{2}} .
$$

\subsection{Benefits: solution to the horizon, flatness, and singularity problems}

VSL theories solve basic problems of standard cosmology such as the flatness problem and the horizon problem. The first one can be coped with, when one inserts (13) into the Friedmann equation (11) to get

$$
\frac{\dot{a}^{2}}{a^{2}}=\frac{8 \pi G_{0} C}{3} a^{-3(w+1)}+\frac{k c_{0}^{2} a^{2 n-2}(2 n-1)}{2 n+3 w+1},
$$

and the density term (with an ansatz for the variability of $c=c_{0} a^{n}$, with $n=$ const, and $C=$ const.) will dominate the curvature term at large scale factor if

$$
2 \geq 2 n+3(w+1) .
$$

The second one is solved bearing in mind that for large scale factor the solution is $a(t)=t^{2 / 3(w+1)}$ and the proper distance to the horizon reads as

$$
d_{H}=c(t) t=c_{0} a^{n}(t) t=c_{0} a_{0}^{n} t^{(3 w+3+2 n) / 3(w+1)}
$$

so that the scale factor grows faster than $d_{H}$ under the same condition as in (15).

Varying constants can also remove ("regularize") or change the nature of singularities within the framework of Friedmann geometry [12]. In Table 1 we see the properties of these singularities. It shows how the enumerated quantities behave at the singularity $t=t_{s}$ : the scale factor $a$, the mass density $\rho$, the pressure $p$, the pressure derivatives, and the barotropic index $w$.

Interesting remarks related to regularizing singularities are as follows:

- In order to regularize an SFS or an FSF singularity by varying $c(t)$, the light should slow and eventually stop propagating at a singularity. This is in analogy to loop quantum cosmology (LQC), where in the anti-newtonian limit $c=c_{0} \sqrt{1-\varrho / \varrho_{c}} \rightarrow 0$ for $\varrho \rightarrow \varrho_{c}$ with $\varrho_{c}$ being the critical density [13]. The low-energy limit $\varrho \ll \varrho_{0}$ gives the standard value $c \rightarrow c_{0}$.) 
- To regularize an SFS or an FSF by varying gravitational constant $G(t)$ - the strength of gravity has to become infinite at an initial (curvature) singularity. Effectively, a new singularity - of strong coupling for a physical field such as $G \propto 1 / \Phi$ appears. Such problems were already dealt with in superstring and brane cosmology where both the curvature singularity and a strong coupling singularity show up [3].

\subsection{Problems: derivation of the field equations from a proper action}

As it was already mentioned, the equations (11)-(13) have just been obtained in a special frame - the one in which $c$ is a constant and does not lead to any extra boundary terms (apart from standard ones). Einstein equations were simply generalized:

$$
G_{\mu \nu}-g_{\mu \nu} \Lambda=\frac{8 \pi G}{\psi} T_{\mu \nu}
$$

while the action (2) varied in a standard way leads to different field equations

$$
G_{\mu \nu}-g_{\mu \nu} \Lambda=\frac{8 \pi G}{\psi} T_{\mu \nu}-\frac{1}{\psi} \psi_{; ; ; \mu}+\frac{1}{\psi} \square \psi
$$

The application of Bianchi identity to (17) gives a conservation equation with dynamical $\psi$

$$
T_{; \mu}^{\mu v}=-T^{\mu v} \psi_{; \mu}
$$

If $\psi$ was supposed to be a dynamical matter field, then one could get the evolution equation using the Lagrangian

$$
L_{\psi}=-\frac{\omega}{16 \pi G \psi} \dot{\psi}^{2}
$$

but working only in a preferred frame and with $\psi$ not coupled to $\sqrt{-g}$. The best formulation was recently proposed by Moffat [18].

\subsection{Benefits of varying $\alpha$ cosmology}

Since one does not brake Lorentz invariance in varying fine structure constant $\alpha$ theories, then there are no such problems in these models - the standard variational principle applies and the dynamical equation for the scalar field is given.

According to the definition, any variability of $c$ (or $e, \hbar)$ is related to the variability of $\alpha$ :

$$
\frac{\Delta \alpha}{\alpha}=-\frac{\Delta c}{c} .
$$

The best constraint on $\Delta \alpha$ which comes from 2 billion years ago is from Oklo natural nuclear reactor and reads as $\Delta \alpha / \alpha=(0.15 \pm 1.05) \cdot 10^{-7}$ at $\mathrm{z}=0.14$. There are other constraints e.g. from VLT/UVES (Very Large Telescope/Ultraviole Echelle Survey) quasars: $\Delta \alpha / \alpha=(0.15 \pm 0.43) \cdot 10^{-5}$ at $1.59<\mathrm{z}<$ 2.92, and from SDSS (Sloan Digital Sky Survey) quasars: $\Delta \alpha / \alpha=(1.2 \pm 0.7) \cdot 10^{-4}$ at $0.16<\mathrm{z}<0.8$.

\section{$2.4 \alpha$-dipole}

According to [19] there is anisotropy in the variability of the fine structure constant in the sky ( $\alpha$ dipole at R.A.17.4 $\pm 0.9 h, \delta=-58 \pm 9$ as measured independently by Keck Telescope $(\Delta \alpha<0)$ and VLT. Some specific measurements of $\alpha$ are listed below (in parts per million; UVES - Ultraviolet and Visual Echelle Telescope, HARPS - High Accuracy Radial velocity Planet Searcher, LP - Large Program measurement): 
Table 2. Measurements of $\alpha$

\begin{tabular}{|c|c|c|c|c|}
\hline Object & $\mathrm{z}$ & $\Delta \alpha / \alpha$ & Spectrograph & Ref. \\
\hline \hline HE0515-4414 & 1.15 & $-0.1 \pm 1.8$ & UVES & Molaro et al. (2008) [20] \\
\hline HE0515-4414 & 1.15 & $0.5 \pm 2.4$ & HARPS/UVES & Chand et al. (2006) [21] \\
\hline HE0001-2340 & 1.58 & $-1.5 \pm 2.6$ & UVES & Agafonowa et al. (2011) [22] \\
\hline HE2217-2818 & 1.69 & $1.3 \pm 2.6$ & UVES-LP & Molaro et al. (2013) [23] \\
\hline Q1101-264 & 1.84 & $5.7 \pm 2.7$ & UVES & Molaro et al. (2008) [20] \\
\hline
\end{tabular}

\subsection{Strongest bound - atomic clock Rosenband bound at $z=0$}

Rosenband [24] measurement gives the following bound at $z=0$ (present)

$$
\left(\frac{\dot{\alpha}}{\alpha}\right)_{0}=(-1.6 \pm 2.3) \times 10^{-17} \mathrm{yr}^{-1}
$$

which can be transformed onto the bound for the scalar field coupling $\xi$ :

$$
\left|\frac{\dot{\alpha}}{\alpha}\right|_{0}=|\xi| H_{0} \sqrt{3 \Omega_{\Phi 0}\left|1+w_{\Phi 0}\right|},
$$

and translates for $H_{0}=(67.4 \pm 1.4) \mathrm{km} \cdot \mathrm{s}^{-1} \mathrm{Mpc}^{-1}$ Planck value) into the conservative $(3 \sigma)$ bound

$$
|\xi| \sqrt{3 \Omega_{\Phi 0}\left|1+w_{\Phi 0}\right|}<10^{-6} .
$$

\section{Redshift drift test of varying $c$ models}

Redshift drift measurement [27] is to collect data from two light cones separated by 10-20 years to look for a change in redshift of a source as a function of time.

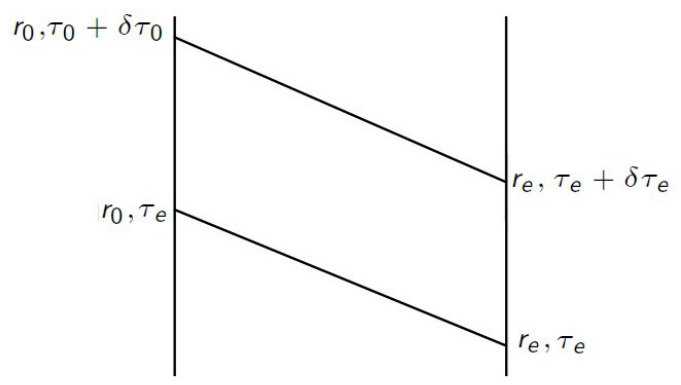

Figure 1. The idea of redshift drift measurement.

There is a relation between the times of emission of light by the source $t_{e}$ and $t_{e}+\Delta t_{e}$ and the times of their observation at $t_{o}$ and $t_{o}+\Delta t_{o}$ which in VSL theory generalizes into [28]

$$
\int_{t_{e}}^{t_{o}} \frac{c(t) d t}{a(t)}=\int_{t_{e}+\Delta t_{e}}^{t_{o}+\Delta t_{o}} \frac{c(t) d t}{a(t)}
$$

and for small $\Delta t_{e}$ and $\Delta t_{o}$ transforms into

$$
\frac{c\left(t_{e}\right) \Delta t_{e}}{a\left(t_{e}\right)}=\frac{c\left(t_{0}\right) \Delta t_{o}}{a\left(t_{o}\right)} .
$$


The definition of redshift in VSL theories remains the same as in standard Einstein relativity i.e. $1+z=a\left(t_{0}\right) / a\left(t_{e}\right)$. Using (26) we have

$$
\frac{\Delta z}{\Delta t_{0}}=\frac{\Delta z}{\Delta t_{0}}(z, n)=H_{0}(1+z)-H(z)(1+z)^{n} .
$$

In the limit $n \rightarrow 0$ the formula (27) reduces to the one of the standard Friedmann universe [27]. Bearing in mind the definitions of dimensionless density parameters $\Omega_{i}$, and assuming flat universe one has

$$
H^{2}(z)=H_{0}^{2}\left[\Omega_{m 0}(1+z)^{3}+\Omega_{\Lambda}\right]
$$

and so (27) gives

$$
\frac{\Delta z}{\Delta t_{0}}=H_{0}\left[1+z-\sqrt{\Omega_{m 0}(1+z)^{3+2 n}+\Omega_{\Lambda}(1+z)^{2 n}}\right]
$$

which can further be rewritten to define a new redshift function

$$
\tilde{H}(z) \equiv(1+z)^{n} H(z)=H_{0} \sqrt{\sum_{i=1}^{i=k} \Omega_{w i}(1+z)^{3\left(w_{e f f}+1\right)}},
$$

where $w_{\text {eff }}=w_{i}+\frac{2}{3} n$. The redshift drift can be measured by future telescopes such as E-ELT (European-Extremely Large Telescope), TMT (Thirty Meter Telescope), GMT (Giant Magellan Telescope) as well as gravitational wave detectors (DECIGO) DECi-Hertz Interferometer Gravitational Wave Observatory and BBO (Big Bang Observer).

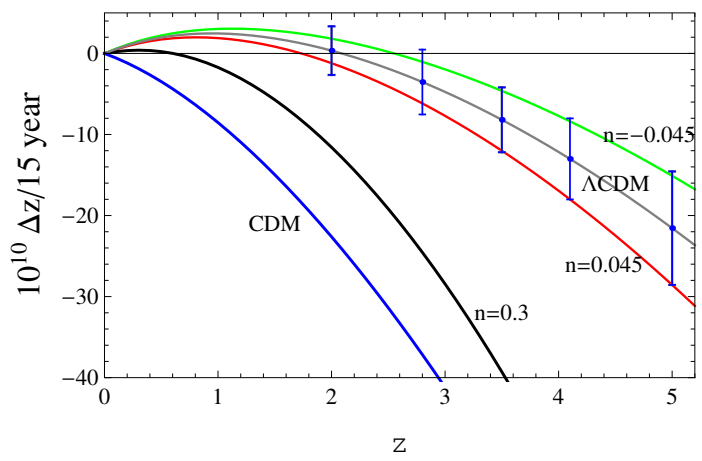

Figure 2. The redshift drift effect for 15 year period of observations for various values of the varying speed of light parameter $n$. The error bars are taken from [29] and presumably show that for $|\mathrm{n}|<0.045$ one cannot distinguish between VSL models and $\Lambda \mathrm{CDM}$ models.

This relation is presented in Fig. 2 from which one cas easily see that for small values of the parameter $n$ (small variation of $c$ ) the dark energy can be mimicked while for large values of $n$ there is a clear distinction between dark energy which can be detected.

\section{Measuring $c$ by future galaxy surveys}

Speed of light $c$ appears in many observational quantities. Among them in the angular diameter distance [33]

$$
D_{A}=\frac{D_{L}}{(1+z)^{2}}=\frac{a_{0}}{1+z} \int_{t_{1}}^{t_{2}} \frac{c(t) d t}{a(t)}
$$


where $D_{L}$ is the luminosity distance, $a_{0}$ present value of the scale factor (normalized to $a_{0}=1$ later), and we have taken the spatial curvature $k=0$ (otherwise there would be sin or sinh in front of the integral). Using the definition of redshift and the dimensionless parameters $\Omega_{i}$ we have

$$
D_{A}=\frac{1}{1+z} \int_{0}^{z} \frac{c(z) d z}{H(z)}
$$

where

$$
H(z)=\sqrt{\Omega_{r 0}(1+z)^{4}+\Omega_{m 0}(1+z)^{3}+\Omega_{\Lambda}} .
$$

\subsection{Angular diameter distance maximum}

Due to the expansion of the universe, there is a maximum of the distance at

$$
D_{A}\left(z_{m}\right)=\frac{c\left(z_{m}\right)}{H\left(z_{m}\right)},
$$

which can be obtained by simple differentiating (32) with respect to $z$ :

$$
\frac{\partial D_{A}}{\partial z}=-\frac{1}{(1+z)^{2}} \int_{0}^{z} \frac{c(z) d z}{H(z)}+\frac{1}{1+z} \frac{c(z)}{H(z)}=0 .
$$

In a flat $k=0$ cold dark matter (CDM) model, there is a maximum at $z_{m}=1.25$ and $D_{A} \approx 1230 \mathrm{Mpc}$. For the standard $\Lambda \mathrm{CDM}$ model of our interest the maximum is at $1.4<z_{m}<1.8$. The product of $D_{A}$ and $H$ gives exactly the speed of light $c$ at maximum (the curves intersect at $z_{m}$ ):

$$
D_{A}\left(z_{m}\right) H\left(z_{m}\right)=c_{0} \equiv 299792.458 \mathrm{kms}^{-1}
$$

if we believe it is constant (defined officially by Bureau International des Poids et Mesures (BIPM) [30] and a relative error is claimed to be $\left.10^{-9}[31]\right)$.
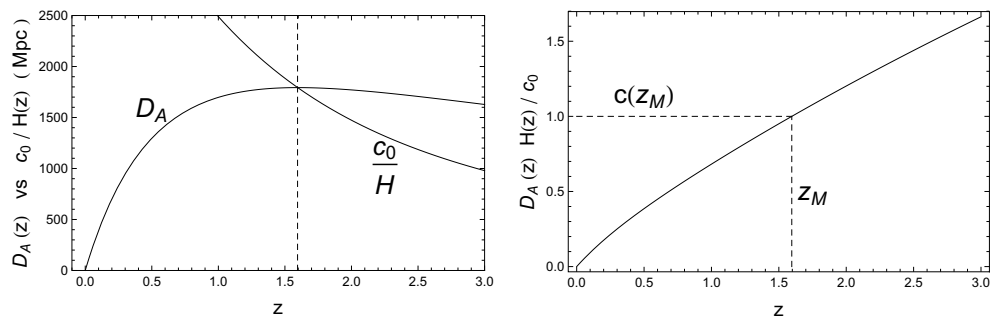

Figure 3. $D_{A}$ and $H(z)$ crossing plots at $z_{m}$.

Measuring $z_{m}$ is problematic if one uses $D_{A}$ only (this is because of a large plateau around $z_{m}$ which makes it difficult to avoid errors from small sample of data - besides, one has binned data, observational errors, and intrinsic dispersion). However, one can appeal to an independent measurement of $c_{0} / H(z)$ which is the radial (line-of-sight) mode of the baryon acoustic oscillations (BAO) surveys for which $D_{A}(z)$ is the tangential mode [32]. In other words, we have both tangential and horizontal modes as

$$
y_{t}=\frac{D_{A}}{r_{s}}, \quad y_{r}=\frac{c}{H r_{s}},
$$


where

$$
r_{s}=\int_{z_{\text {dec }}}^{\infty} \frac{c c_{s}(z) d z}{H(z)}
$$

is the sound horizon size at decoupling and $c_{s}$ the speed of sound. The measurements of BAO are from BOSS DR11 CMASS [34]

$$
\frac{D_{V}}{r_{s}\left(z_{d}\right)}=13.85 \pm 0.17 \quad \text { at } \quad \bar{z}=0.57
$$

where the volume-averaged distance is

$$
D_{V}=\left[(1+z)^{2} c z \frac{D_{A}^{2}}{H}\right]^{\frac{1}{3}}
$$

and from BOSS DR11 LOWZ [35]

$$
D_{V}=(1264 \pm 25)\left(\frac{r_{s}\left(z_{d}\right)}{r_{s, f i d}\left(z_{d}\right)}\right) \quad \text { at } \quad \bar{z}=0.32 \text {. }
$$

\subsection{The method to measure $c$.}

In Ref. [33] a new method to measure $c$ based on the relation (35) was proposed, and it is composed of the following steps:

1. Independently measuring $D_{A}(z)$ and $H(z)$.

2. Calculating $z_{m}$.

3. Getting the product $D_{A}\left(z_{m}\right) H\left(z_{m}\right)=c\left(z_{m}\right)$.

4. Calculating $\Delta c=c\left(z_{m}\right)-c_{0}$, first assuming that $c\left(z_{m}\right)$ may not be equal to $c_{0}$.

5. Determining possible level of variability/constancy of $c$.

For this sake the background $\Lambda \mathrm{CDM}$ model with an ansatz [36]

$$
c(a) \propto c_{0}\left(1+\frac{a}{a_{c}}\right)^{n}
$$

is taken into account, where $a_{c}$ is the scale factor at the transition epoch from some $c(a) \neq c_{0}$ (at early times) to $c(a) \rightarrow c_{0}$ (at late times to now). Three scenarios are considered [33]:

1) standard case $c=c_{0}$;

2) $a_{c}=0.005, n=-0.01 \rightarrow \Delta c / c \approx 1 \%$ at $z \propto 1.5$;

3) $a_{c}=0.005, n=-0.001 \rightarrow \Delta c / c \approx 0.1 \%$ at $z \propto 1.5$. After using $10^{3}$ Euclid project [37] mock data simulations [38], one obtains the following results:

1) $z_{m}=1.592_{-0.039}^{+0.043}$ (fiducial model input $z_{m}=1.596$ ) and $c / c_{0}=1 \pm 0.009$;

2) $z_{m}=1.528_{-0.036}^{+0.038}$ (fiducial $z_{m}=1.532$ ) and $c\left(z_{m}\right) / c_{0}=1.00925 \pm 0.00831$; and

$$
<c\left(z_{m}\right) / c_{0}-1 \sigma_{c\left(z_{m}\right) / c_{0}}>=1.00094_{-0.00033}^{+0.00014},
$$

so that a possible detection by Euclid of $1 \%$ variation at $1 \sigma$-level in future will be possible.

3) $z_{m}=1.584_{-0.039}^{+0.042}$ (fiducial $z_{m}=1.589$ ) and $c\left(z_{m}\right) / c_{0}=1.00095 \pm 0.00852$ and

$$
<c\left(z_{m}\right) / c_{0}-1 \sigma_{c\left(z_{m}\right) / c_{0}}>=0.99243_{-0.00013}^{+0.00016},
$$

so that a detection by Euclid of $0.1 \%$ variation at $1 \sigma$-level will not be possible. 


\subsection{Other surveys and perspectives}

In fact, Euclid will have 1/10 of the error bars of the current missions like WiggleZ Dark Energy Survey (e.g. [39]). Other missions which will be competitive to Euclid and useful for our task will be: Dark Energy Spectroscopic Instrument (DESI) [40]; Square Kilometer Array (SKA) [41]; Wide-Field Infrared Survey Telescope (WFIRST) [42] (especially having largest sensitivity at potential $z_{m}$ region i.e. $1.5<\mathrm{z}<1.6)$.

\section{Conclusions}

Varying speed of light $c$ (and related to them varying fine structure constant $\alpha$ ) theories which attract more interest among physicists have both advantages as well as problems. The advantages of them is that they solve the flatness, horizon problems, and in some special cases, the singularity problem. However, their violation of Lorentz invariance leads to a choice of a preferred frame and a drop of standard variational principle. On the other hand, $\alpha$-varying theories have better formulation and due to the definition of $\alpha$, they can be related to varying- $c$ theories.

In this paper we have proposed some new tests to check variability of $c$ in future telescope/space missions. The first was the redshift drift test which gives clear prediction for redshift drift effect which can potentially be measured by future telescopes like E-ELT, TMT, GMT, DECIGO/BBO. The second was to use baryon acoustic oscillations test and the Hubble function test to independently measure the radial $D_{A}$ and tangential mode $c / H$ of the volume distance $D_{V}$ at the angular diameter distance maximum $z_{m}$.

Putting this last method in simple terms we have considered a "cosmic" measurement of the speed of light $c$ with $D_{A}$ giving the dimension of length playing the role of a "cosmic ruler" and $1 / H$ giving the dimension of time playing the role of a "cosmic clock"/"chronometer" i.e.

$$
c=\frac{D_{A}}{\left(\frac{1}{H}\right)} .
$$

We have checked that $1 \%$ variability of $c$ can be tested at $1 \sigma$ level by Euclid mission. It is likely that such variability will also be possible to test by SKA and WFIRST.

\section{Acknowledgements}

The research of V.S., M.P.D., and A.B. was supported by the Polish National Science Center Grant DEC-2012/06/A/ST2/00395.

\section{References}

[1] P.A.M. Dirac, Nature 139, 323 (1937); Proc. Roy. Soc. A165, 189 (1938).

[2] C.H. Brans, R.H. Dicke, Phys. Rev. 124, 925 (1961).

[3] J. Polchinski, String Theory (Cambridge University Press, Cambridge, 1998).

[4] A. Einstein, Jahrbuch für Radioaktivität und Elektronik 4, 411 (1907).

[5] R.H. Dicke, Rev. Mod. Phys. 29, 363 (1957).

[6] J.-P. Petit, Mod. Phys. Lett. A 3, 1527 (1988).

[7] J. Moffat, Int. J. Mod. Phys. D 2, 351 (1993).

[8] A. Albrecht, J. Magueijo, Phys. Rev. D59, 043516 (1999).

[9] J.K. Webb et al. Phys. Rev. Lett. 87, 091301 (2001).

[10] J.D. Barrow, H. Sandvik, J. Mageuijo, Phys. Rev. D65, 063504 (2002). 
[11] J.D. Barrow, D. Kimberly, J. Magueijo, Class. Quantum Grav. 21, 4289 (2004).

[12] M.P. Da̧browski, K. Marosek, Journ. Cosmol. Astrop. Phys. 02, 012 (2013).

[13] T. Cailleteau, J. Mielczarek, A. Burrau, and J. Grain, Class. Quantum Grav. 29, 095010 (2012).

[14] M.P. Dąbrowski, K. Marosek, A. Balcerzak, Mem. Soc. Astron. Ital. 85, 44-49 (2014).

[15] S. Nojiri, S.D. Odintsov and S. Tsujikawa, Phys. Rev. D 71,063004 (2005).

[16] M.P. Da̧browski, T. Denkiewicz, AIP Conference Proceedings 1241, 561 (2010).

[17] M.P. Dąbrowski, Mathematical Structures of the Universe, M. Eckstein, M. Heller, S.J. Szybka (eds.), (Copernicus Center Press, Kraków), 99 (2014).

[18] J.W. Moffat, arXiv: 1501.01872.

[19] J.K. Webb et al., Phys. Rev. Lett. 107, 191101 (2011).

[20] P. Molaro, D. Reimers, I. I. Agafonova, S. A. Levshakov, Eur. Phys. JST 163, 173 (2008).

[21] H. Chand, R. Srianad, P. Petitjean, B. Aracil, R. Quast and D. Reimers, Astron. Astroph. 451, 45 (2006).

[22] I. I. Agafonova, P. Molaro and S. A. Levshakov, Astron. Astroph. 529, A28 (2011).

[23] P. Molaro et al., Astron. Astroph. 555, A68 (2013).

[24] T. Rosenband et al., Science 319, 1808 (2008).

[25] T. Denkiewicz, M.P. Da̧browski, C.J.A.P. Martins, P. Vielzeuf - Phys. Rev. D 89, 083514 (2014).

[26] M.P. Dąbrowski, T. Denkiewicz, C.J.A.P. Martins, P. Vielzeuf - Phys. Rev. D 89, 123512 (2014).

[27] A. Sandage, Astrophys. J. 136, 319 (1962); A. Loeb, Astrophys. J. 499, L11 (1998).

[28] A. Balcerzak, M.P. Da̧browski, PLB 728, 15 (2014).

[29] C. Quercellini et al., Phys. Rep. 521, 95 (2012).

[30] www.bipm.org

[31] K.M. Evenson, Phys. Rev. Lett. 29, 1346 (1972).

[32] S. Nesseris, L. Perivolaropoulos, Phys.Rev. D73, 103511 (2006).

[33] V. Salzano, M.P. Dạbrowski, R. Lazkoz, Phys. Rev. Lett. 114, 101304 (2015).

[34] L. Samushia et al., Mon. Not. R. Astron. Soc. 439, 3504 (2014).

[35] R. Tojeiro et al., arXiv: 1401.1768.

[36] J. Magueijo, Rep. Prog. Phys. 66, 2025 (2003).

[37] L. Laureijs et al. 0912.0914 (Euclid Collaboration).

[38] A. Font-Ribeira et al., Journ. Cosmol. Astropart. Phys. 05, 023 (2014).

[39] B.D. Sherwin, arXiv: 1207.4543.

[40] M. Levi et al., Astroph. Journ. 803, 21 (2015).

[41] Ph. Bull et al., 1405.1452)

[42] D. Spergel et al., arXiv: 1305.5425. 